Article

\title{
Soft Ion Sputtering of PAni Studied by XPS, AFM, TOF-SIMS, and STS
}

\author{
Christopher M. Goodwin ${ }^{1, *}$, Zachary E. Voras ${ }^{2}$, Xiao Tong ${ }^{3}$ and Thomas P. Beebe Jr. ${ }^{4}$ \\ 1 Department of Physics, Stockholm University, 10691 Stockholm, Sweden \\ 2 Department of Chemistry, West Chester University of Pennsylvania, West Chester, PA 19383, USA; \\ zvoras@wcupa.edu \\ 3 Brookhaven National Laboratory Center for Functional Nanomaterials, Upton, New York, NY 11973, USA; \\ XTong@bnl.gov \\ 4 Department of Chemistry and Biochemistry Newark, University of Delaware, Newark, DE 19716, USA; \\ Beebe@udel.edu \\ * Correspondence: Christopher.Goodwin@fysik.su.se
}

Received: 3 September 2020; Accepted: 7 October 2020; Published: 12 October 2020

\begin{abstract}
Herein is a study of the soft sputtering method, gas cluster ion sputtering (GCIS), and its effects on the atomic, morphologic, and band structure properties of polyaniline (PAni) as studied with X-ray photoelectron spectroscopy (XPS), time-of-flight secondary ion mass spectrometry, atomic force microscopy, and scanning tunneling spectroscopy (STS). The GCIS source used was a 1000 argon atom cluster with $4 \mathrm{keV}$ energy, which resulted in a sputter yield of $3.4 \pm 0.2 \times 10^{-3} \mathrm{~nm}^{3}$ per argon atom. Soft ion sputtering reduced the sample by explicitly removing the oxidized contaminants as determined by surface sensitive techniques: XPS and Time-of-flight secondary ion mass spectrometry (TOF-SIMS). By the use of STS we found that by removing the oxidized components, an overall shift of electronic states occurred, transitioning the states closer to the Fermi edge by $0.3 \mathrm{~V}$.
\end{abstract}

Keywords: conductive polymer; GCIS; band structure; density of states; surface treatment; surface; TOF-SIMS; soft materials; soft coating

\section{Introduction}

Conductive polymers have an array of applications due to being organic semiconductors [1], which allow for simpler and less costly preparation methods compared to typical crystalline semiconductors. Polyaniline (PAni), in particular, is of interest since the electronic structure can be easily tailored with the addition of various salts. PAni has found applications as antistatic coatings [2], capacitors [3], anti-corrosion coatings [4,5], and molecular sensors [6,7]; for many of these operations, surface roughness and composition are critical. There have been a wide variety of methods that have been used to make PAni surfaces (with varying surface roughness), including evaporation deposition [8], spin coating [9], drop-casting [10], electrodeposition [11], and DC sputtering [6]. Though PAni has many industrial uses to date, few research studies have focused on how to modify these surfaces once prepared. The lack of surface studies of conductive polymers is most likely due to the complexity of preparing such a sample for ultra-high vacuum measurements.

PAni can have its conductivity controlled by changing the ratio of the two forms on nitrogen; the three general types of PAni base are shown in Figure 1. The leucoemeraldine seen in Figure 1a is the completely reduced form, emeraldine has a 50:50 mixture of the reduced and oxidized form of the nitrogen shown in Figure 1b, and finally (per)nigraniline is the fully oxidized form Figure $1 c$. The ratio of the imine nitrogen shown in red compared to amine nitrogen shown in blue determines the type of PAni made and conductivity. Herein the emeraldine base form was studied. 
a<smiles>CC1C=CC(=Nc2ccc(N=C3C=CC(=Nc4ccc(N(C)C)cc4)C=C3)cc2)C=C1</smiles>

Figure 1. Atomic model of the various forms of Polyaniline (PAni), leucoemeraldine (a), emeraldine (b), and (per)nigraniline (c). The two types of nitrogen are amine in blue and imine in red; the ratio of these two forms dictate the kind of PAni.

One of the main obstacles in using PAni is the presence of oxidized species that limit conduction [12]. The surface contamination has limited the study of polymers such as PAni by traditional vacuum techniques. The recent development of gas cluster ion sputtering (GCIS) has allowed for the use of sputtering on soft materials, a process previously impossible due to the large number of damaged molecules left from the impinging ions. GCIS has been shown to remove surface contaminations without modifying the chemical composition of the exposed substrate [13,14] the effect that GCIS has on surface properties has been mainly unstudied. Towards this end GCIS was applied to remove the oxidized layer which contaminates the surface of PAni and show the changing speciation of the sample by sputtering. Furthermore, it is also shown is the changes to surface roughness as a result of GCIS and how in-plane (Zalar) rotation changes the topologic changes caused by sputtering. Finally, the changes to the density of states of the conductive polymer are shown. By these results it is shown that PAni surfaces can be prepared by non-vacuum means and recover the surface properties that are of interest by cleaning with GCIS.

There has been a push to understand both experimental and theoretical perspectives of soft sputtering. Molecular dynamics (MD) has modeled gas clusters interactions with surfaces $[15,16]$ to form a theoretical understanding. Experimental GCIS research has compiled the sputter yields for various materials, including Irganox 1010, which has become the standard for GCIS [17], consumer polymers such as polypropylene [18], polystyrene [19], mixed polymers [20], as well as complex biologic samples such as brain tissue of a rat [13]. A common consideration with these studies was to understand general trends in sputter yield and to determine a "universal sputtering curve" [15,21]. To understand what can affect the sputter rate, multiple parameters including angle [22], cluster mass, and kinetic energy [21], and even comparing argon-based clusters to other gas species cluster compositions [23] have been studied and compared. Yet, there has been little work on understanding the dynamics of how GCIS modifies the morphology of the samples. Irganox 1010 has been used as it is a literature-acceptable standard for soft sputtering to establish the GCIS rate [24]. Several studies have been published on how the surface structure is changed by cluster sputtering or how cluster sputtering changes the morphology of other polymers. There have been studies that investigated the effects of GCIS on the morphology of mixed polymer blends [25], sputter source [26,27], and sample rotation [28]. 
A wide variety of methods to change polymers' surface roughness and composition have been attempted, including solvents [29,30], hot press [31], graft treatments [32,33], plasma [34], and abrasion [35]. While all of these methods are useful in many situations, there does not yet exist a simple process to prepare a pristine polymeric surface in a vacuum system. As mentioned above, some have developed methods to deposit polymeric films in a vacuum, yet these have limitations. These vacuum deposition methods often leave the vacuum system irreversibly damaged since the polymer will deposit on the vacuum chamber walls. A far more reliable and straightforward way to prepare a pristine polymer surface would be to be able to sputter the material, as is often done for hard materials. With the judicious application of GCIS, it is possible to remove the topmost layers from a polymer film and have a fresh surface to investigate. There remain several concerns beyond simple chemical changes to the surface. Herein it is investigated what effect soft sputtering has on the chemical, morphologic, and electronic composition of PAni.

\section{Materials and Methods}

The samples were prepared in a similar manner as Abdulrazzaq et al. [36]; a 5 by weight percent solution of $20 \mathrm{k}$ Da emeraldine base polyaniline was dissolved in N-Methyl-2-pyrrolidone (NMP). All chemicals were purchased from Sigma Aldrich (St. Louis, MO, USA). To dissolve the PAni powder, the mixture was vortexed for $5 \mathrm{~min}$, then sonicated for $30 \mathrm{~min}$, followed by centrifuging at $50 \mathrm{~Hz}$ for $30 \mathrm{~min}$ to isolate the undissolved polymer. The undissolved component is mostly attributed to the oxidized polymer. The supernatant solution was spin-cast onto indium tin oxide (ITO) film at $500 \mathrm{rpm}$ with a Nanocs (New York, NY, USA). The cleaning process for the ITO coated glass slide substrate followed a standard method [36], which was solicitation in $10 \mathrm{w} / \mathrm{w} \%$ sodium hydroxide for $3 \mathrm{~min}$ and then rinsed with deionized water (18.2 Mohm). This cleaning process was repeated twice before a final solicitation in isopropyl alcohol (IPA) for $5 \mathrm{~min}$. The slides were then left in IPA until the polymer was spin-coated onto the slides. The back of the glass slides (uncoated) were patterned by hand to orient the surface before spin-coating and facilitated tracking the measurement positions on the sample; this was done so that a direct comparison of the morphologic changes could be determined.

All atomic force microscope (AFM) images were gathered with a Multimode IV (currently Bruker, formerly Digital Instruments, Camarillo, CA, USA) AFM. To determine the GCIS effect directly, AFM images were gathered at the same position on the film before and after sputtering; this was done by using the pattern on the back of the glass slide to orient the sample as well as micron-scale details on the surface, see reference [28] for more information. Imaging the same spot was crucial to analyze the morphological changes as a result of GCIS directly. Silicon tips were purchased from Bruker Nano (Camarillo, CA, USA), and all AFM images were gathered in tapping mode; the AFM tips had a nominal frequency of $300 \mathrm{kHz}$, and a tip radius of approximately $7 \mathrm{~nm}$ (VTESPA-300).

To determine the chemical composition, X-ray photoelectron spectroscopy (XPS) was used, GCIS was carried out within the same system, a Thermo Scientific (East Grinstead, UK) K-alpha+ housed at the Surface Analysis Facility (SAF) at the University of Delaware (Newark, DE, USA). To reduce X-ray damage, a small X-ray spot size of $100 \mu \mathrm{m}$ was used, care was taken to avoid extended and unnecessary $\mathrm{X}$-ray exposure. This was done by ensuring the sample was not illuminated by $\mathrm{X}$-rays while measurements were not being gathered. A low energy electron flood gun was used to reduce any charging effects that may have accumulated. Monochromatic X-rays were used, with aluminum K-alpha source at a photon energy of $1486.7 \mathrm{eV}$. Argon gas clusters of nominal 1000 atoms were utilized with a kinetic energy of $4 \mathrm{keV}$ and were used for all experiments. The ion beam used had a nominal spot size of $300 \mu \mathrm{m}$ at $60^{\circ}$ relative to the sample surface. The sputter source was rastered over the sample surface in a rectangle shape, $1.85 \mathrm{~mm}$ by $3.69 \mathrm{~mm}$, at speeds of $333 \mathrm{~Hz}$ and $3 \mathrm{~Hz}$ respectively using a nonsynchronous square wave pattern. To preform Zalar rotation, a motorized gear assembly was used, and Zalar rotation was performed at a rate of $0.04 \mathrm{~Hz}$.

To determine more detailed information on the atomic structure of the surface, Time-of-flight secondary ion mass spectrometry (TOF-SIMS) in the SAF at the University of Delaware was carried 
out using a TOF-SIMS V system (ION-TOF GmbH; Munster, Germany). All TOF-SIMS measurements were carried out in "static" mode with $\mathrm{Bi}_{3}^{+}$ions using a total ion dosage of $10^{12}$ per $\mathrm{cm}^{2}$ at an ion current of $0.30 \mathrm{pA}$ and a cycle time of $0.10 \mathrm{msec}$. A bismuth/manganese alloy ion source was used to form the bismuth ions in a "high-current bunched mode" with an ion beam of energy $25 \mathrm{keV}$ and a bunch width less than $1 \mathrm{~ns}$. All TOF-SIMS negative-ion mode spectra gathered with a typical mass resolution of $6000 \mathrm{M}$ per $\Delta \mathrm{M}$ (determined at ${ }^{30} \mathrm{Si} \mathrm{m} / \mathrm{z}$ ), all spectra were calibrated to the characteristic ranging from mass 1 to $96 \mathrm{AMU}\left(\mathrm{H}^{-}, \mathrm{C}^{-}, \mathrm{CH}^{-}, \mathrm{CH}_{2}^{-}, \mathrm{CH}_{3}^{-}, \mathrm{C}_{2}^{-}, \mathrm{C}_{3}^{-}, \mathrm{C}_{4}^{-}, \mathrm{C}_{5}^{-}, \mathrm{C}_{6}^{-}, \mathrm{C}_{7}^{-}\right.$, and $\left.\mathrm{C}_{8}^{-}\right)$.

Scanning tunneling spectroscopy (STS) was carried out using multiprobe UHV-STM/XPS/UPS/ISS/LEED/TPD system at the Center for Functional Nanomaterials (CFN), which is a U.S. DOE Office of Science Facility, at Brookhaven National Laboratory. STS spectra were acquired with an SPM 1000 (RHK, Troy, MI, USA) at room temperature with a base pressure of $2.6 \times 10^{-10}$ mbar using custom-built electronics and GXSM software (version 2.0). Tungsten tips were electrochemically prepared and in situ sputter-cleaned for all STS spectra. All bias values are in reference to the bias applied to the sample. All STS results from samples were prepared in the SAF as described above, then transported to CFN. To ensure limited surface oxidation, samples were prepared and sealed in a vacuum suitcase with a base pressure of $1.3 \times 10^{-7}$ mbar. For each STS spectrum, measurements were repeated nine times, and the resulting data were averaged by a third-order boxcar average. To calculate the derivative, the Savitzky-Golay method was used. The voltage of the sample was scanned at 0.02 volts per second, with 1100 data points for each scan, resulting in data points taken every $5.19 \mathrm{mV}$.

The PAni films were measured to be $69.9 \pm 0.3 \mathrm{~nm}$ thick as measured by AFM in the method described elsewhere [28,37]. The samples were then thinned to $59.3 \pm 0.5 \mathrm{~nm}$ by GCIS. The sputtering beam was rastered over an area of $3.69 \mathrm{~mm}$ by $1.85 \mathrm{~mm}$ using a non-synchronous square wave pattern. The long dimension was scanned at a rate of $333 \mathrm{~Hz}$, and the short direction was at $3 \mathrm{~Hz}$. The argon cluster beam spot size is an elliptical circle with a profile of $300 \pm 100 \mu \mathrm{m}$ positioned; the ion source is $60^{\circ}$ to sample normal. When the sample was rotated, it was at a rate of $0.1 \mathrm{~Hz}$; care must be taken to ensure the center of rotation is the center of the sputter location. The sputter rate was determined to be $3.4 \pm 0.2 \times 10^{-3} \mathrm{~nm}^{3}$ per argon atom and was independent of whether the sample was rotated or not.

\section{Results}

\subsection{XPS}

Figure 2a depicts the effects of sample preparation and sputtering on PAni as a powder film and sputtered film, as seen by the carbon region by XPS. The topmost spectrum shows the carbon 1s region of the powder as provided mounted on carbon tape. The middle spectrum shows a sample of PAni after spin casting the polymer, as described earlier. By comparing the top spectra to the mid spectra, it can be seen that there was no significant change in this carbon 1s as a result of making PAni films. The bottom spectrum was the same sample and position as in the spectra middle spectra after soft sputtering. Figure $2 b$ shows the nitrogen 1 s XPS region of the same samples; Figure $2 c$ shows the oxygen 1s XPS region of the previously mentioned samples; the oxygen is attributed to contamination of the polymer as Prokes et al. [12] described.

As seen in Table 1, the carbon atomic percent of the sample started at $85.2 \pm 0.4 \%$ as a powder to $84.9 \pm 0.3 \%$ as a spin-cast film to $86.7 \pm 0.2 \%$ after sputtering. The nitrogen atomic percent of the samples started with $11.7 \pm 0.5 \%$ as a powder to $12.2 \pm 0.3 \%$ when spin-cast to $12.6 \pm 0.2 \%$ after sputtering. The oxygen atomic percent decreases as a result of sputtering from $3.1 \pm 0.4 \%$ as a powder to $2.9 \pm 0.5 \%$ as the thin film was made to $0.8 \pm 0.6 \%$ after sputtering. 


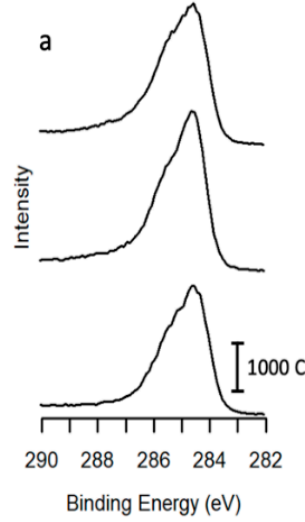

C $1 s$

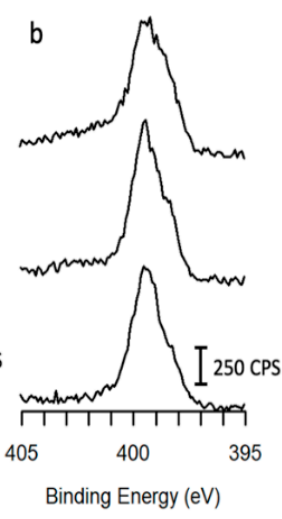

$\mathrm{N}$ 1s
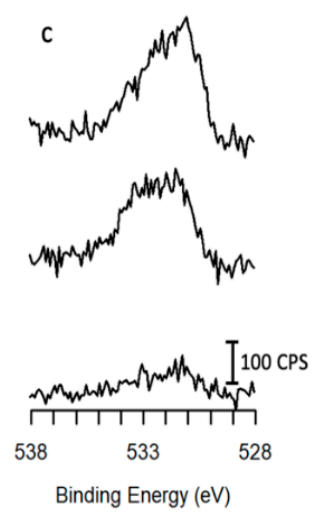

$01 s$

Figure 2. XPS spectra of PAni at various points during sample modification. The top line in each set of spectra is the pure PAni powder as received from the manufacturer. The middle line shows the sample after preparation and spin-coated onto indium tin oxide (ITO) (there was no signal from the indium of the underlayer of the ITO). The last set of spectra is of the thin film after gas cluster ion sputtering (GCIS) to identify the oxygen source. The polymer film was not made so thin as to reveal the ITO substrate. Therefore, the oxygen signal would be only from PAni and not the substrate. (a) shows the carbon 1s spectra; (b) is the nitrogen 1s spectra; $(\mathbf{c})$ is the oxygen $1 \mathrm{s.}$

Table 1. Measured atomic percent composition (all errors are standard deviations with $n=3$ ). See text for speciation atomic percentages.

\begin{tabular}{cccc}
\hline Sample & Carbon & Nitrogen & Oxygen \\
\hline Powder & $85.2 \pm 0.4 \%$ & $11.7 \pm 0.5 \%$ & $3.2 \pm 0.4 \%$ \\
Film & $84.9 \pm 0.3 \%$ & $12.2 \pm 0.3 \%$ & $2.9 \pm 0.5 \%$ \\
Post sputter & $86.7 \pm 0.2 \%$ & $12.6 \pm 0.2 \%$ & $0.8 \pm 0.6 \%$ \\
Bulk values $^{1}$ & $86 \%$ & $14 \%$ & - \\
\hline \multicolumn{4}{c}{ Based on atomic structure. }
\end{tabular}

Nitrogen spectra were fit with three distinct nitrogen components and a Shirley background, as seen in Figure 3a. The lowest binding energy specie is $(398.4 \mathrm{eV})$ and is attributed to the amine nitrogen shown as the blue nitrogen in Figure 1, the next peak is located at $399.6 \mathrm{eV}$ and is the imine and the red nitrogen in Figure 1. The final nitrogen component at $400.8 \mathrm{eV}$ is an oxidized nitrogen that was a contaminant in the polymer. The oxidized nitrogen is most likely a mixture of several types of nitrogen, including $\mathrm{NO}_{x}$ species and some amount of emeraldine salt [38]. The ratio of imine to amine remains nearly constant at approximately three times more imine than the amine. The third nitrogen decreases as the sample was sputtered, falling from $3.3 \%$ in the powder to $3.1 \%$ in the film to $1.1 \%$ after sputtering.

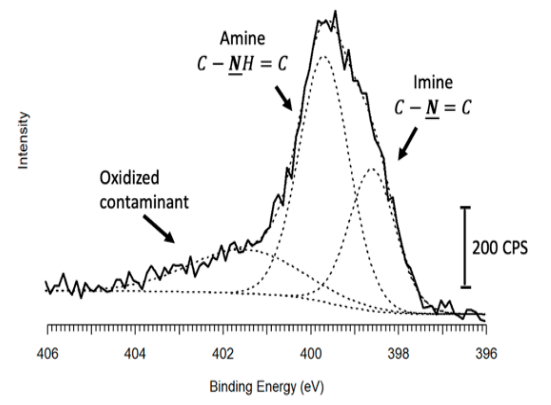

b

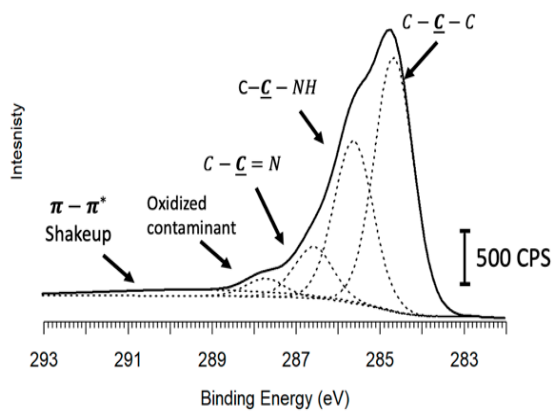

Figure 3. XPS spectra fit before sputtering of the nitrogen 1s (a) and carbon 1s (b). 
By peak fitting the carbon 1s region with a shirley background, five separate states of carbon are identified, as seen in Figure 3. The most reduced form of carbon is the carbonaceous carbon calibrated to be at $284.6 \mathrm{eV}$, and the carbon bound to the amine nitrogen appears next at $285.6 \mathrm{eV}$ - the carbon at $286.6 \mathrm{eV}$ the carbon bound to the imine nitrogen. An oxidized contaminant appears at $287.7 \mathrm{eV}$, which is likely a carbon bound to oxygen twice, such as ketones or aldehydes or to oxygen and nitrogen. The final component is the $\pi-\pi^{*}$ shake-up peak caused by the aromatic rings, the peak of which appears as a broad and small component centered at $290.8 \mathrm{eV}$. The ratio between the peaks agrees very well with the expected values. The oxidized carbon component peak at $287.7 \mathrm{eV}$, which is most likely contaminants, decreases in intensity as the sample was sputtered, from $2.8 \%$ to $1.8 \%$ after sputtering.

The oxygen present in Figure 2 is caused by ambient oxidation of the polymer; this is why the oxygen is present in the powder as well as on the thin film. The oxygen region is not peak fit due to the multitude of possible peaks with little evidence for any specific state; the region is most likely a set of alcohol, ketone, and nitrate peaks; it is possible that some of these species exist as a salt with the oxidized nitrogen described above. The oxygen atomic percent decreases as the sample was prepared and more so after sputtering. The atomic oxygen percent in the powder was measured to be $3.1 \%$. Spin-casting the polymer slightly reduced the oxygen content to $2.9 \%$, and sputtering further reduced the oxidized component to $0.8 \%$. As described above, the oxygen is attributed to ambient oxidation; this occurs on any exposed portion of the polymer. The decrease in oxygen from the powder to the thin film is due to there being less surface area for the thin film than the powder. The oxidized portion of the polymer in the powder is less soluble. It is therefore not deposited during spin casting, resulting in a polymer film with a bulk composition very similar to the idealized polymer. This decrease in oxygen due to each step in preparation correlates with the declining signal of the most highly oxidized nitrogen component. The net change in the sample as a result of GCIS was a reduction of the oxygen-containing portion of the thin film. Any oxygen in PAni is widely considered to hinder conduction, either as disrupting the polymer network or as a salt, which shifts the electron density of the film [39].

\section{2. $A F M$}

Each thin film sample was imaged before and after soft sputtering with atomic force microscopy (AFM) to determine the surface morphology changes as a result of GCIS. Care was taken to image the same spot before and after sputtering. All images shown in Figure 4 are of a $4 \times 4 \mu \mathrm{m}^{2}$ area. Figure $4 \mathrm{a}$,c show the sample surface of two samples before sputtering. Figure $4 \mathrm{~b}$ shows the same spot as in Figure 4a after sputtering; this sample was not rotated during sputtering. Figure $4 \mathrm{~d}$ shows the same place as in Figure $4 \mathrm{c}$ after sputtering (for this sample, Zalar rotation was used) [40].

Through comparing Figure $4 \mathrm{a}, \mathrm{b}$, there can be seen the formation of striations, which are widely known to be reduced by rotating the sample in-plane, known as Zalar rotation. The detail in the upper right was imaged so that the position on the sample can be tracked. All morphology maps are on a $35 \mathrm{~nm}$ height scale, leaving the large features in the upper right off the height scale. The striations seen in Figure $4 \mathrm{~b}$ are in line with the sputter gun and are seen sporadically in the published literature that uses sputtering when samples are not rotated. Though the large polymeric defects in the upper right of Figure 4 were maintained through sputtering, the striations and islands in Figure $4 \mathrm{~b}$,d do also cover the entirety of the large defect. Figure $4 \mathrm{c}$, d show the direct effects of Zalar rotation on surface morphology. The sputter lines that were observed in Figure $4 \mathrm{~b}$ were not seen in $4 \mathrm{~d}$; this clearly shows the importance of Zalar rotation can have during GCIS. The surface had an observable change as a result of sputtering even through the rotation. The resulting island pattern was on the nanometer-scale and was smaller in width and height than the striation pattern seen in Figure $4 b$. As in Figure $4 a, b$, a large defect in the polymer surface was used to track the measurement position. As with the striation pattern of Figure $4 b$, the island pattern was also observed on the off-scale feature. 

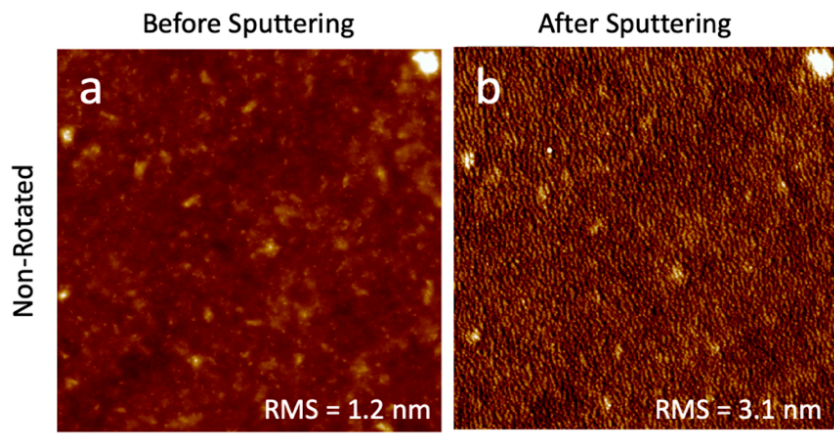

$35 \mathrm{~nm}$
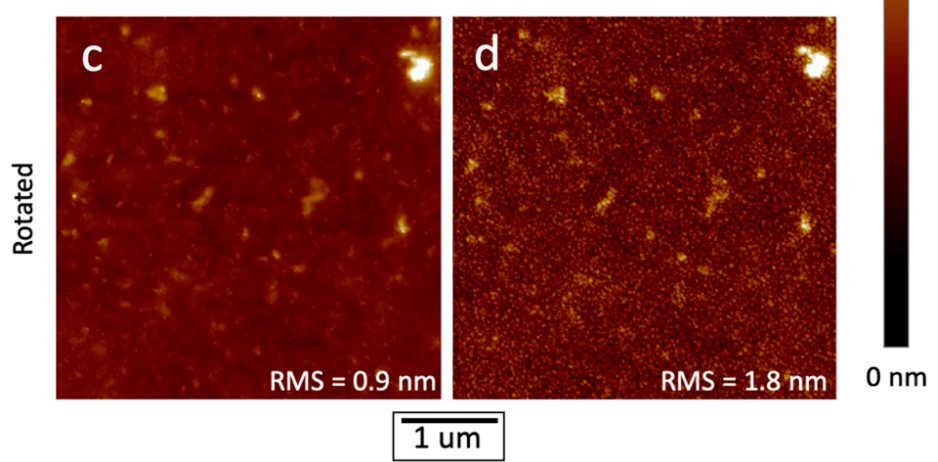

Figure 4. Atomic force microscopy (AFM) images of the thin films (a,c) before sputtering, (b) after sputtering with a stationary sample, and (d) after sputtering with Zalar (in-plane) sample rotation. AFM images are at the same positions before and after sputtering, meaning $(\mathbf{a}, \mathbf{b})$ are the same area of the sample, as well as (c,d). The surface defect that in the upper-right corner was included in the images to show any offset between the corresponding sets of AFM images. The height scale bar on the right applies to all AFM images.

Figure 5 displays the sets of pixel height distributions based on the AFM images of Figure 4. Within Figure 5, the solid line represents the data from Figure 4a or Figure 4c, samples before sputtering. The dashed line represents the image of Figure $4 \mathrm{~b}$. Finally, the symbol " $\bullet$ " is based on Figure $4 \mathrm{~d}$. By comparing the different distributions of height, the quantification of changes can be made. Figure $5 \mathrm{a}$ is the direct comparison of height distribution caused by GCIS from the sample that was not rotated; the solid line is before sputtering from Figure $4 \mathrm{a}$, and the dashes are after sputtering, Figure $4 \mathrm{~b}$. Figure $5 \mathrm{~b}$ shows the same comparison while the sample is rotated, which is of the height distribution from AFM images in Figure 4c,d shown as a solid line and "•". Finally, Figure 5c makes a direct comparison between the results that rotation has on root-mean-square (RMS) roughness caused by sputtering. As before, the dashed line represents the data from Figure $4 \mathrm{~b}$, the sample that was held stationary while being sputtered; the "•" shows the data from Figure $4 \mathrm{~d}$, the sample that was rotated in-plane during sputtering.
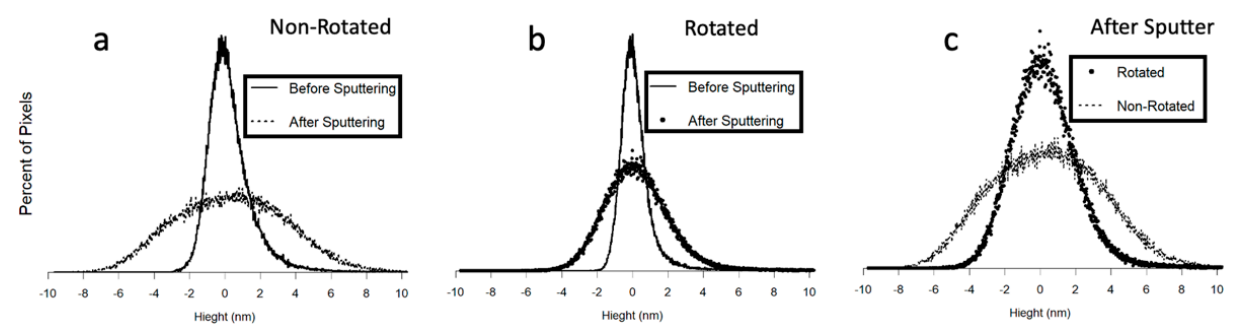

Figure 5. Zero centered pixel height distributions of AFM images from Figure 4 showing before and after sputtering a non-rotated sample (a); before and after sputtering, a rotated sample (b); and after sputtering a rotated and non-rotated sample (c). The root-mean-square (RMS) values in Figure 4 are the same as the standard deviations of the distributions here in Figure 4. 
From Figure 5, comparisons between samples before and after GCIS can be made, as well as the effects of stationary sputter and Zalar rotation during sputtering. By comparing the height distributions, the net effects of soft sputtering on roughness, and the role that in-plane rotation had in surface morphology can be deduced. Due to surface inhomogeneities, the two samples began with different RMS roughness to start with, in part due to the relatively small scale of the images, but also due to the inherent nature of spin-casting polymers yielding an inconsistent surface roughness. Figure $5 \mathrm{a}-\mathrm{c}$ had been set so that the maximum of the distribution is $0.0 \mathrm{~nm}$ to show the change in distributions widths. From Figure 5a, it can be seen that the distribution of sample height was broadened, the RMS is $1.2 \pm 0.1 \mathrm{~nm}$ for the sample before sputtering, the distribution was broadened to $3.1 \pm 0.1 \mathrm{~nm}$ as a result of sputtering an increase of $1.9 \mathrm{~nm}$. Figure $5 \mathrm{~b}$ shows the impact that sputtering had on sample roughness showing the RMS starts as $0.9 \pm 0.1 \mathrm{~nm}$ before sputtering and increases to $1.8 \pm 0.1 \mathrm{~nm}$ when the sample was sputtered while being rotated, a change of $0.9 \mathrm{~nm}$. Finally, Figure $5 \mathrm{c}$ shows the distributions of samples that have been sputtered; from this, it was seen that the stationary sample had a more significant deviation by $1.3 \mathrm{~nm}$ as compared to the rotated sample. The change in roughness shows that rotation can reduce the roughening caused by GCIS.

\subsection{TOF-SIMS}

TOF-SIMS spectra shown in Figure 6 are normalized to the total ion count. Figure 6 shows the mass spectrum around nominal mass $62 \mathrm{AMU} / \mathrm{z}$; the solid line is of a sample before sputtering, and the dashed line is the sample after soft sputtering. The peaks are $\mathrm{NO}_{3}^{-}$at $61.988 \mathrm{AMU}, \mathrm{HCNOF}^{-}$ at 62.004 $\mathrm{AMU}$, and $\mathrm{C}_{5} \mathrm{H}_{2}^{-}$at 61.016 $\mathrm{AMU}$. The decrease in nitrate signal agrees with the previous XPS results, which showed a reduction in the most oxidized nitrogen components. As well, the XPS results on oxygen content also showed a reduced signal with sputtering. Though peak amplitude is related to abundancy, it is also related to ease of ionization; this is why the $\mathrm{HCNOF}^{-}$peak amplitude appears so large, but no fluorine is detected by XPS. The relative change in peak amplitude is exclusively the effect of abundancy, therefore showing that GCIS has a large impact on oxidized components over other species. Overall, the TOF-SIMS spectra were in good agreement with previous studies [41].

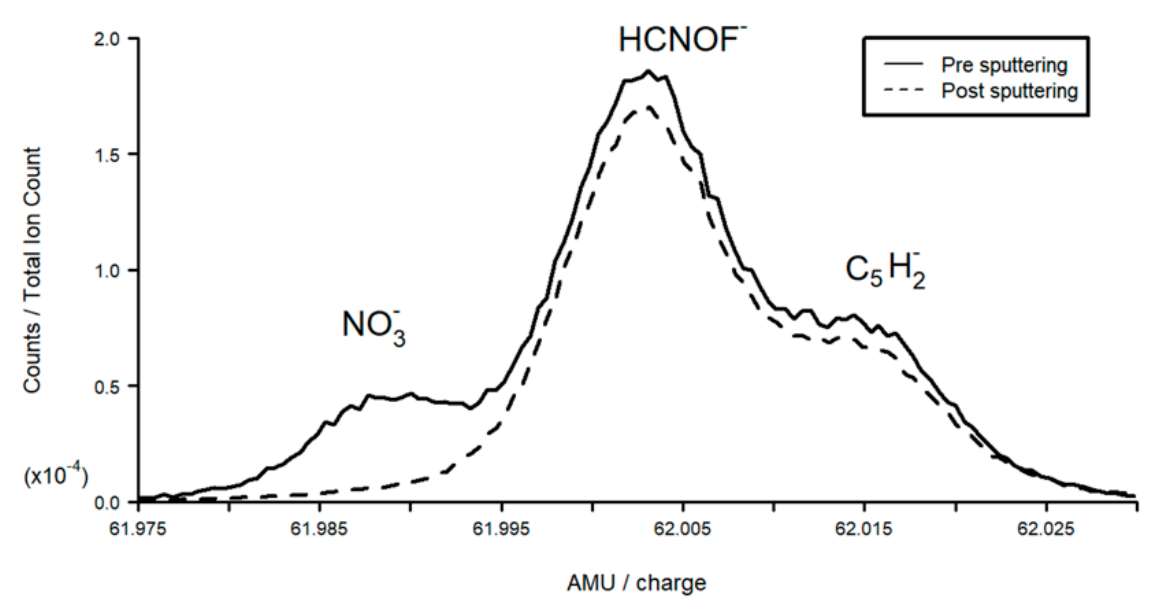

Figure 6. Time-of-flight secondary ion mass spectrometry (TOF-SIMS) spectra of the mass range about 62 AMU, which shows the removal of the $\mathrm{NO}_{3}^{-}$signal due to GCIS.

\subsection{STS}

Scanning tunneling spectroscopy (STS) was performed to understand the direct effect that soft sputtering had on the band structure of the PAni. Figure 7 shows the direct effect that soft sputtering had on the PAni films. Figure 7a shows the data of a thin film that which not sputtered; Figure $7 \mathrm{~b}$ shows the sample after GCIS without Zalar rotation. While both sets of data have some similarities and differences, the slopes of the two data sets far from the fermi ends, before -0.75 and beyond 
0.75 volts, are within statistical variance, and the measurements are to be considered the same. There is a notable change in the states closest to the Fermi edge. As seen in the states from -0.75 to 0.0 volts, the DOS intensity shifts closer to 0.0 volts, the fermi edge. The change is the surface is attributed to the removal of oxygen and oxidized contaminants, which appears to somewhat remediate the electronic structure. The shift in states is a change of 0.3 volts towards the Fermi edge, which may result in more conductivity. The structure of the STS spectra is in agreement with published studies of PAni, specifically bulk and doped polyaniline $[42,43]$. Surface conductivity is dictated by the surface DOS, as seen by Figure 7, GCIS has shift the surface DOS to be nearer the Fermi edge, likely increasing surface conductivity.
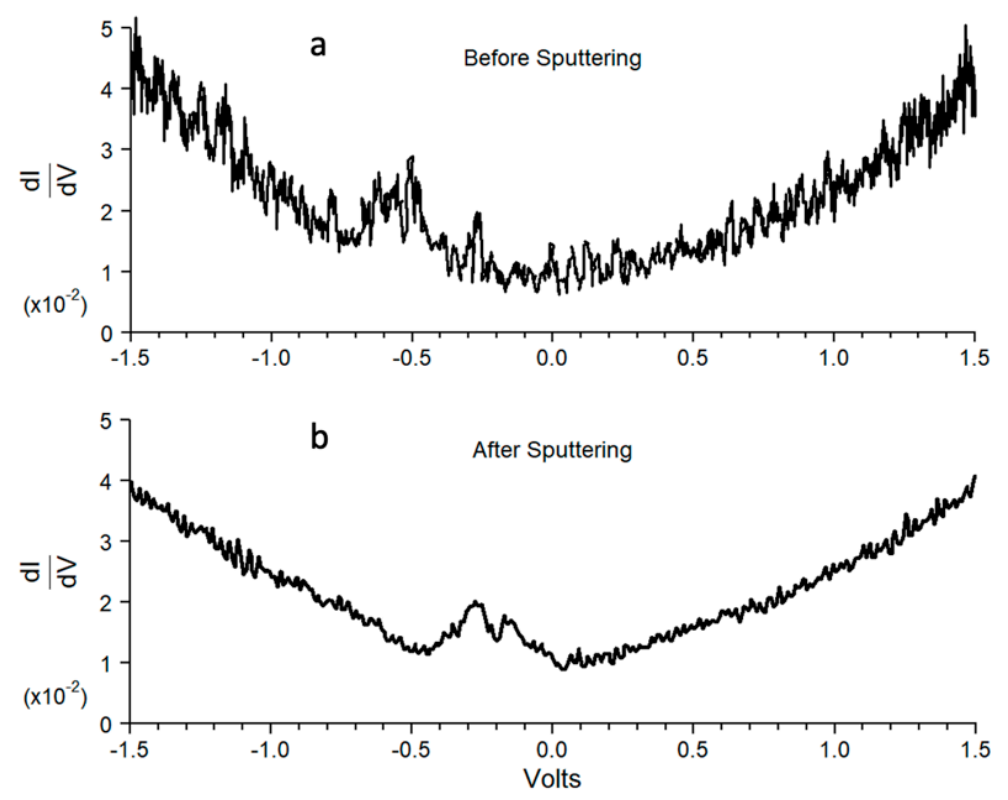

Figure 7. Direct measurements of the DOS, both occupied and unoccupied, about the Fermi edge by scanning tunneling spectroscopy (STS). The top STS spectra (a) are of a sample that was not sputtered while the bottom spectra, (b) are of a sample after GCIS.

\section{Conclusions}

GCIS had a large effect on the atomic, chemical, and morphologic structure of PAni films. Through sputtering, the surface oxygen-containing components, oxidized contaminants, were selectively removed or lowered to less than that of the untreated polymeric powder or thin film. The unprocessed polymer powder started with 3.1 atomic percent oxygen, the thin film had $2.9 \%$ oxygen, yet sputtering reduced the oxygen percentage to $0.8 \%$. The sputter yield of PAni with $4 \mathrm{keV}$ clusters of 1000 atom argon at was $3.4 \pm 0.2 \times 10^{-3} \mathrm{~nm}^{3}$ per argon atom. The morphology of the thin film was significantly modified by soft sputtering with the roughness (RMS of the height) increasing by nearly a factor of 3 from $1.2 \mathrm{~nm}$ to $3.1 \mathrm{~nm}$ while sputtering with a stationary sample, and by a factor of 2 to $1.8 \mathrm{~nm}$ when the sample was rotated.

The change in surface roughness by soft sputtering could have implications on some analysis of the film films. Though sputtering made the surface rougher, the large decrease in surface roughness by Zalar rotation would have a significant effect on depth resolution during depth profiles performed on this (and perhaps chemically similar) polymers. The dynamic sputtering effect should not be ignored when analyzing layered structures. By removal of the contaminated polymer species, specifically, the oxidized nitrogen, an overall shift in occupied states between -0.75 and 0.0 eV towards the Fermi edge of the surface occurred, which may have increased surface conductivity.

GCIS has been shown to be a vacuum surface treatment for PAni that remediates the oxidative effect of ambient conditions, including those inherent to the polymer as a powder in its crude state. By using GCIS, it is now able to reduce the polymer to a chemical state more similar to the bulk 
structure. Thereby, making it is possible to study the surface of the pristine polymer by typical surface analysis techniques. Furthermore, it was shown that it was possible to study PAni without the need for wet chemical preparations within the vacuum chamber. We hope that as GCIS becomes more common interesting polymers, such as conductive polymers, may become more widely studied by surface analysis.

Along with opening the possibility of surface analysis, vacuum deposition methods to prepare layered structures containing PAni may benefit from GCIS treatment due to the reducing effects of GCIS. When conductive polymers are often incorporated into electronics, conductivity is paramount. Herein we have shown a way to possibly improve the conductivity of PAni, which would have a sizeable downstream effect on the viability of polymer-based electronics.

Author Contributions: C.M.G. designed the experiment, gathered and processed data, and wrote the manuscript. Z.E.V. gathered and analyzed data as well as editing the manuscript. X.T. provided experimental resources. T.P.B.J. supervised, provided funding, developed the experimental concept, and edited the manuscript. All authors have read and agreed to the published version of the manuscript.

Funding: This research was funded by NSF (9724307) and NIH NIGMS COBRE (P30-GM110758) for support of the Surface Analysis Facility at the University of Delaware. This research used resources of the Center for Functional Nanomaterials, which is a U.S. DOE Office of Science Facility, at Brookhaven National Laboratory under Contract No. DE-SC0012704.

Conflicts of Interest: The authors declare no conflict of interest.

\section{References}

1. MacDiarmid, A.G. "Synthetic metals": A novel role for organic polymers (Nobel lecture). Angew. Chem. Int. 2001, 40, 2581-2590. [CrossRef]

2. Soto-Oviedo, M.A.; Araújo, O.A.; Faez, R.; Rezende, M.C.; de Paoli, M.-A. Antistatic coating and electromagnetic shielding properties of a hybrid material based on polyaniline/organoclay nanocomposite and EPDM rubber. Synth. Met. 2006, 156, 1249-1255. [CrossRef]

3. Bélanger, D.; Ren, X.; Davey, J.; Uribe, F.; Gottesfeld, S. Characterization and Long-Term Performance of Polyaniline-Based Electrochemical Capacitors. J. Electrochem. Soc. 2000, 147, 2923-2929. [CrossRef]

4. Talo, A.; Passiniemi, P.; Forsén, O.; Yläsaari, S. Polyaniline/epoxy coatings with good anti-corrosion properties. Synth. Met. 1997, 85, 1333-1334. [CrossRef]

5. Kraljić, M.; Mandić, Z.; Duić, L. Inhibition of steel corrosion by polyaniline coatings. Corros. Sci. 2003, 45, 181-198. [CrossRef]

6. Menegazzo, N.; Boyne, D.; Bui, H.; Beebe, T.P., Jr.; Booksh, K.S. DC Magnetron Sputtered Polyaniline-HCl Thin Films for Chemical Sensing Applications. Anal. Chem. 2012, 84, 5770-5777. [CrossRef] [PubMed]

7. Menegazzo, N.; Herbert, B.; Banerji, S.; Booksh, K.S. Discourse on the utilization of polyaniline coatings for surface plasmon resonance sensing of ammonia vapor. Talanta 2011, 85, 1369-1375. [CrossRef]

8. Adhikari, S.; Banerji, P. Enhanced conductivity in iodine doped polyaniline thin film formed by thermal evaporation. Thin Solid Films 2010, 518, 5421-5425. [CrossRef]

9. Cao, Y.; Treacy, G.M.; Smith, P.; Heeger, A.J. Solution-Cast Films of Polyaniline-Optical-Quality Transparent Electrodes. Appl. Phys. Lett. 1992, 60, 2711-2713. [CrossRef]

10. Shimano, J.Y.; MacDiarmid, A.G. Polyaniline, a dynamic block copolymer: Key to attaining its intrinsic conductivity? Synth. Met. 2001, 123, 251-262. [CrossRef]

11. Nguyen, T.D.; Camalet, J.-L.; Lacroix, J.-C.; Aeiyach, S.; Pham, M.C.; Lacaze, P.-C. Polyaniline electrodeposition from neutral aqueous media: Application to the deposition on oxidizable metals. Synth. Met. 1999, 102, 1388-1389. [CrossRef]

12. Prokes, J.; Trchova, M.; Hlavata, D.; Stejskal, J. Conductivity ageing in temperature-cycled polyaniline. Polym. Degrad. Stab. 2002, 78, 393-401. [CrossRef]

13. Bich, C.; Havelund, R.; Moellers, R.; Touboul, D.; Kollmer, F.; Niehuis, E.; Gilmore, I.S.; Brunelle, A. Argon Cluster Ion Source Evaluation on Lipid Standards and Rat Brain Tissue Samples. Anal. Chem. 2013, 85, 7745-7752. [CrossRef] [PubMed]

14. Winograd, N. The magic of cluster SIMS. Anal. Chem. 2005, 77, 142A-149A. [CrossRef] 
15. Paruch, R.J.; Postawa, Z.; Garrison, B.J. Seduction of Finding Universality in Sputtering Yields Due to Cluster Bombardment of Solids. Acc. Chem. Res. 2015, 48, 2529-2536. [CrossRef] [PubMed]

16. Delcorte, A.; Debongnie, M. Macromolecular Sample Sputtering by Large Ar and CH4 Clusters: Elucidating Chain Size and Projectile Effects with Molecular Dynamics. J. Phys. Chem. C 2015, 119, 25868. [CrossRef]

17. Shard, A.G.; Ray, S.; Seah, M.P.; Yang, L. VAMAS interlaboratory study on organic depth profiling. Surf. Interface Anal. 2011, 43, 1240-1250. [CrossRef]

18. Williams, D.F.; Abel, M.-L.; Grant, E.; Hrachova, J.; Watts, J.F. Flame treatment of polypropylene: A study by electron and ion spectroscopies. Int. J. Adhes. Adhes. 2015, 63, 26-33. [CrossRef]

19. Bernasik, A.; Haberko, J.; Marzec, M.M.; Rysz, J.; Łużny, W.; Budkowski, A. Chemical stability of polymers under argon gas cluster ion beam and x-ray irradiation. J. Vac. Sci. Technol. B 2016, 34, 30604. [CrossRef]

20. Seah, M.P.; Havelund, R.; Shard, A.G.; Gilmore, I.S. Sputtering Yields for Mixtures of Organic Materials Using Argon Gas Cluster Ions. J. Phys. Chem. B 2015, 119, 13433. [CrossRef]

21. Seah, M.P. Universal Equation for Argon Gas Cluster Sputtering Yields. J. Phys. Chem. C 2013, 117, 12622-12632. [CrossRef]

22. Seah, M.P.; Spencer, S.J.; Shard, A.G. Angle Dependence of Argon Gas Cluster Sputtering Yields for Organic Materials. J. Phys. Chem. B 2015, 119, 3297-3303. [CrossRef] [PubMed]

23. Shard, A.G.; Havelund, R.; Spencer, S.J.; Gilmore, I.S.; Alexander, M.R.; Angerer, T.B.; Aoyagi, S.; Barnes, J.-P.; Benayad, A.; Bernasik, A.; et al. Measuring Compositions in Organic Depth Profiling: Results from a VAMAS Interlaboratory Study. J. Phys. Chem. B 2015, 119, 10784. [CrossRef]

24. Shard, A.G.; Havelund, R.; Seah, M.P.; Spencer, S.J.; Gilmore, I.S.; Winograd, N.; Mao, D.; Miyayama, T.; Niehuis, E.; Rading, D.; et al. Argon Cluster Ion Beams for Organic Depth Profiling: Results from a VAMAS Interlaboratory Study. Anal. Chem. 2012, 84, 7865-7873. [CrossRef]

25. Taylor, A.J.; Graham, D.J.; Castner, D.G. Reconstructing accurate ToF-SIMS depth profiles for organic materials with differential sputter rates. Analyst 2015, 140, 6005-6014. [CrossRef]

26. Chu, Y.-H.; Liao, H.-Y.; Lin, K.-Y.; Chang, H.-Y.; Kao, W.-L.; Kuo, D.-Y.; You, Y.-W.; Chu, K.-J.; Wu, C.-Y.; Shyue, J.-J. Improvement of Gas Cluster Ion Beam- (GCIB)-Based Molecular Secondary Ion Mass Spectroscopy (SIMS) Depth Profile with $\mathrm{O}_{2}{ }^{+}$Cosputtering. Analyst 2016, 141, 2523. [CrossRef]

27. You, Y.-W.; Chang, H.-Y.; Lin, W.-C.; Kuo, C.-H.; Lee, S.-H.; Kao, W.-L.; Yen, G.-J.; Chang, C.-J.; Liu, C.-P.; Huang, C.-C.; et al. Molecular dynamic-secondary ion mass spectrometry (D-SIMS) ionized by co-sputtering with $\mathrm{C}_{60}{ }^{+}$and $\mathrm{Ar}^{+}$. Rapid Commun. Mass Spectrom. 2011, 25, 2897-2904. [CrossRef] [PubMed]

28. Goodwin, C.M.; Voras, Z.E.; Beebe, T.P. Gas-cluster ion sputtering: Effect on organic layer morphology. J. Vac. Sci. Technol. A 2018, 36, 051507. [CrossRef]

29. Strawhecker, K.E.; Kumar, S.K.; Douglas, J.F.; Karim, A. The critical role of solvent evaporation on the roughness of spin-cast polymer films. Macromolecules 2001, 34, 4669-4672. [CrossRef]

30. Sehgal, T.; Rattan, S. Synthesis, characterization and swelling characteristics of graft copolymerized isotactic polypropylene film. Int. J. Polym. Sci. 2010, 2010. [CrossRef]

31. Doroudiani, S.; Park, C.B.; Kortschot, M.T. Effect of the crystallinity and morphology on the microcellular foam structure of semicrystalline polymers. Polym. Eng. Sci. 1996, 36, 2645-2662. [CrossRef]

32. Bhattacharya, A.; Misra, B.N. Grafting: A versatile means to modify polymers-Techniques, factors and applications. Prog. Polym. Sci. 2004, 29, 767-814. [CrossRef]

33. Yoshida, W.; Cohen, Y. Topological AFM characterization of graft polymerized silica membranes. J. Membr. Sci. 2003, 215, 249-264. [CrossRef]

34. Coburn, J.W. Surface Processing with Partially Ionized Plasmas. IEEE Trans. Plasma Sci. 1991, 19, 1048-1062. [CrossRef]

35. Lanauze, J.A.; Myers, D.L. Ink Adhesion on Corona-Treated Polyethylene Studied by Chemical Derivatization of Surface Functional Groups. J. Appl. Polym. Sci. 1990, 40, 595-611. [CrossRef]

36. Abdulrazzaq, O.; Bourdo, S.E.; Saini, V.; Bairi, V.G.; Dervishi, E.; Viswanathan, T.; Nima, Z.A.; Biris, A.S. Optimization of the Protonation Level of Polyaniline-Based Hole-Transport Layers in Bulk-Heterojunction Organic Solar Cells. Energy Technol. 2013, 1, 463-470. [CrossRef]

37. Ton-That, C.; Shard, A.G.; Bradley, R.H. Thickness of spin-cast polymer thin films determined by angle-resolved XPS and AFM tip-scratch methods. Langmuir 2000, 16, 2281-2284. [CrossRef] 
38. Sreedhar, B.; Sairam, M.; Chattopadhyay, D.K.; Mitra, P.P.; Rao, D.V.M. Thermal and XPS studies on polyaniline salts prepared by inverted emulsion polymerization. J. Appl. Polym. Sci. 2006, 101, 499-508. [CrossRef]

39. Furukawa, Y.; Ueda, F.; Hyodo, Y.; Harada, I.; Nakajima, T.; Kawagoe, T. Vibrational-Spectra and Structure of Polyaniline. Macromolecules 1988, 21, 1297-1305. [CrossRef]

40. Zalar, A. Improved Depth Resolution by Sample Rotation during Auger-Electron Spectroscopy Depth Profiling. Thin Solid Films 1985, 124, 223-230. [CrossRef]

41. Chan, H.S.O.; Ang, S.G.; Ho, P.K.H.; Johnson, D. Static Secondary Ion Mass-Spectrometry (Sims) of Polyanilines-A Preliminary-Study. Synth. Met. 1990, 36, 103-110. [CrossRef]

42. Narayanan, T.N.; Jose, S.; Thomas, S.; Al-Harthi, S.H.; Anantharaman, M.R. Fabrication of a quantum well heterostructure based on plasma polymerized aniline and its characterization using STM/STS. J. Phys. D Appl. Phys. 2009, 42, 165309. [CrossRef]

43. Hassanien, A.; Gao, M.; Tokumoto, M.; Dai, L. Scanning tunneling microscopy of aligned coaxial nanowires of polyaniline passivated carbon nanotubes. Chem. Phys. Lett. 2001, 342, 479-484. [CrossRef]

(C) 2020 by the authors. Licensee MDPI, Basel, Switzerland. This article is an open access article distributed under the terms and conditions of the Creative Commons Attribution (CC BY) license (http://creativecommons.org/licenses/by/4.0/). 